\title{
Replenishment behavior in sequential supply chains
}

\author{
Amin Kaboli ${ }^{1} \quad$ Rémy Glardon $^{1} \quad$ Nicolas Zufferey $^{2} \quad$ Naoufel Cheikhrouhou $^{3}$ \\ ${ }^{1}$ Laboratory for Production Management and Processes, Swiss Federal Institute of Technology at Lausanne \\ (EPFL), CH-1015 Lausanne, Switzerland, amin.kaboli@ gmail.com, remy.glardon@epfl.ch \\ ${ }^{2}$ GSEM - Geneva School of Economics and Management, University of Geneva, Boulevard du Pont-d'Arve 40, \\ 1211 Geneva 4, Switzerland, n.zufferey@unige.ch \\ ${ }^{3}$ Geneva School of Business Administration, University of Applied Sciences Western Switzerland (HES-SO), \\ 1227 Carouge, Switzerland, naoufel.cheikhrouhou @ hesge.ch
}

\begin{abstract}
Inventory managers do not predominantly follow normative optimization models. At best, they introduce a level of bounded rationality in their inventory replenishment decisions. This paper examines the behavior of inventory decision-makers under continuous review in a decentralized supply chain, using an experimental approach with unknown market demand and local information availability. The analysis reveals that not only the magnitude and the variability of order quantity tend to be larger, but also that the order-time intervals is lengthen and highly variable while moving upstream along the supply chain. The role of the inventory managers' replenishment decisions on the echelon holding, backorder, and total costs, is also investigated. Finally, a normative model is designed and its solutions are compared to the experimental results. It is observed that humans do not operate in a perfectly optimal way, but are generally reluctant to risk increasing backorder costs and reducing inventory carrying cost, even if this would lead to lower total cost.

Keywords: Behavioral operations management, Continuous inventory replenishment, Supply chain, Multiechelon network, Laboratory experiment, Inventory management, Cost computation, Optimization, Normative model, Simulation.
\end{abstract}

\section{Introduction}

Consider a decentralized supply chain in which products move through multiple serial echelons before they are shipped to the end customer. In this multi-echelon inventory system, inventory managers make inventory replenishment decisions based on local information (Arguilaget et al., 2017) to regulate the inventory levels, minimize costs, and satisfy downstream customer demand. The inventory replenishment decision is in response to two fundamental questions: how much to order (order quantity) and when to order (order time)?

The frequency of inventory replenishment decisions is determined by the inventory review process, which can be periodic or continuous. In the former, inventory is monitored periodically and inventory replenishment decision is made at a fixed time interval, whereas in the latter, inventory is checked continuously and the order time interval is variable. This fact denotes that in a continuous review system, both order quantity and order time intervals are variable. Numerous periodic and continuous inventory review models have been proposed to optimize echelon's cost and profit, and consequently to improve supply chain performance (e.g., Aviv, 2002; Clark and Scarf, 1960; De Bodt and Graves, 1985; Federgruen and Zipkin, 1984; Silver and Zufferey, 2011). Some works have also proposed to improve the global performance of a supply chain when focusing on the improvement of: the production level only (Thevenin et al., 2015, 2016); the quality management and the value chain (Voldrich et al., 2017); the inventory dispatching when facing unreliable suppliers (Respen et al., 2017); the design of the supply chain itself (Carle et al., 2012). However, the actual behavior shows that decision maker's mind has a restricted capacity to formulate complex problems in a finite time, based on the available information (e.g., Loch and Wu, 2007; Gino and Pisano, 2008; Simon, 1969).

There are considerable empirical and experimental evidences indicating that the actual behavior 
of an inventory manager tends to deviate from the optimal decisions proposed by the extant literature on operations management (e.g., Bolton and Katok 2005, Croson and Donohue 2006, Schweitzer and Cachon 2000, Sterman 2000). One of well-acknowledged operations management problem is the mismatch arising between demand and supply processes. To match demand and supply, inventory managers place orders and regulate inventory levels to avoid backlog. Yet, their efforts are not always successful and most often result in the demand-supply mismatch, which could further exacerbate order quantity and/or order frequency amplification along the chain. Despite of the vast amount of literature on order quantity oscillation and amplification, there is scarce evidence of the order-time interval variation along the supply chain. Given that, interesting questions arise, namely, (i) How do order-time intervals change along the supply chain? (ii) Do all inventory managers behave similarly in different echelons? (iii) How the variability of order quantity and order time intervals influences the costs within the supply chain?

This research aims to understand the ordering behavior of an inventory manager under continuous review on decentralized supply chain. Our experiment shows that not only the magnitude and variability of order quantity increase, but also the order time intervals lengthen and their variability increases along the supply chain from retailer and wholesaler to distributor. Section 2 provides a literature review. Sections 3 and 4 respectively describe the problem and the proposed method. The results are presented in Section 5. A normative model is designed in Section 6 , followed by a discussion in Section 7. Section 8 concludes the paper.

\section{Literature review}

From pivotal studies of the 1960s to the recent ones, researchers have recognized the importance of order amplification in supply chains. The literature in inventory replenishment can be divided into two streams. In the analytical stream (Subsection 2.1), researchers model inventory replenishment and prescribe optimized policies. In the experimental stream (Subsection 2.2), researchers assess inventory replenishment decision and describe the causes of its amplification along the supply chain. As it is the case for this study, even if some papers appear below as experimental (e.g., Lee et al., (1997)), it does not mean that there is no normative model or analytical approach for the involved problem, and vice-versa.

\subsection{Analytical literature stream on inventory replenishment}

Simon (1952) utilizes control theory to analyze simple production-inventory systems. In his work, he highlights pure delays in production-inventory systems that are hard to deal with. To approximate pure delays, he uses exponential delays by smoothing the output signal over time. Vassian (1955) extends Simon's continuous-time theory to discrete time models. In one of the early operations research models addressing multi-echelon inventory, Clark and Scarf (1960) propose optimal policies for a multi-echelon inventory problem under periodic review, when the demand is uncertain. Schmidt and Nahmias (1985) extend the Clark-Scarf approach by characterizing the optimal inventory policy for a simple assembly system. Federgruen and Zipkin (1984) also extend the Clark-Scarf approach from the finite horizon to the infinite horizon. De Bodt and Graves (1985) extend the Clark-Scarf approach from periodic review to continuous review. Badinelli (1992) constructs a model for continuous-review policies in multiechelon supply chain. Grubbström and Wikner (1996) study inventory replenishment systems with the aid of control theory. They show that inventory trigger control policies can be mathematically described by differential equations involving Heaviside and Dirac impulse functions. Chen (1999) considers information delays in decentralized supply chains. He also 
verifies the optimality of implementing a base stock policy under a centralized team. There is no doubt on the importance of models in inventory management and their contribution for finding optimal inventory replenishment decision indicators. But these analytical models assume that people are fully rational, make profit-maximizing decisions, and they are aware of the feedback of their decisions. The actual human behavior shows significant deviations from optimal policies and prescriptive models (e.g., Bendoly et al., 2006; Bendoly et al., 2009; Gino and Pisano, 2008).

\subsection{Experimental literature stream on inventory replenishment}

Forrester (1961) introduces a nonlinear model of supply chain using exponential delays. He designs a role-playing supply-chain simulation game called "Beer distribution game" and shows demand amplification in upstream supply chain. He underlines that structure, policies, and decision makers' interactions in supply chain are the main reasons for order amplification. Towill (1991) and Wikner et al. (1991) extend Forrester's work to two-echelon and threeechelon supply chain contexts, and conclude that the lack of coordination in supply chain leads to variability in order quantity across the supply chain. In an empirical study, Lee et al. (1997) highlight four structural reasons for order quantity amplification: demand signal processing, order batching, price variation, and inventory rationing.

Sterman (1989) presents a generic model of stock management and an ordering decision model based on locally rational heuristics in decentralized supply chains. In fact, by characterizing decision maker mental processes, he bases the decision process on an anchor and adjustment policies, and identifies underweighting the supply line (i.e., units of product ordered but not yet received) as the cause of order quantity amplification. Dogan and Sterman $(2005,2006)$ study phantom ordering in order quantity decision-making processes. Phantom orders arise in supply chains when an upstream supplier is unable to fulfill orders on-time. Thus, the customer responds to backlogs by placing larger order quantities. In a similar work, Oliva and Goncalves (2007) study overreaction in order quantity decision-making processes. As in previous studies, they find that decision makers ignore the supply line and in case of shortage, they saturate order quantity adjustment at a maximum value instead of overreacting. In experimental studies, researchers show that shorter ordering and shipment lead-times improve decentralized supply chain performance under periodic review (e.g., Kaminsky and Simchi-Levi, 1998; Kimbrough et al., 2002; Steckel et al., 2004). Also, sharing point of sale information (e.g., Croson and Donohue, 2003; Gupta et al., 2001; Steckel et al., 2004), using electronic data interchange (Machuca and Barajas, 2004), and communication (Wu and Katok, 2006), can significantly improve decentralized supply chain performance under periodic review. Croson and Donohue (2006) show that order quantity amplification remains even when the market demand is known and when all four structural causes of order quantity amplification are removed. Rong et al. (2008) consider the reactions to supply and demand uncertainty, and introduce reverse order quantity amplification. Croson et al. (2012) investigate the role of coordination stock on supply chain stability, and show that uncertainty of individual decisions is one of the behavioral causes of order quantity amplification.

Despite of the vast literature on order quantity amplification, there is scarce evidence of the order time behavior. Given the evidence about the order quantity amplification along the supply chain, an interesting question arises: how do order time intervals change along the supply chain? To answer to it, we design and run experiments in a decentralized sequential supply chain under continuous inventory replenishment.

\section{Problem description}


Let $i$ be the echelon index ( $i=[R, W, D] ; R$ : retailer, $W$ : wholesaler, $D$ : distributor) in a supply chain, and $i=M$ for the market index. Each inventory manager fulfills the orders received from the downstream customer if sufficient on-hand inventory is available, otherwise $\mathrm{s} / \mathrm{he}$ accumulates backlogs. S/he decides on how much and when to order with the upstream supplier and places orders $O Q^{i}$ at time intervals $O T^{i}$.

Market demand is unknown and customer orders are transferred in the form of information flows from market to factory, trigging material flows from upstream to downstream. Both information and material flows are subject to delays: order lead-time $\left(L_{o}=1\right.$ day $)$, and shipment lead-time ( $L_{s} \geq 2$ days). The nominal shipment lead-time is two days. The shipment lead-time is potentially variable and depends on the availability of on-hand inventory at the upstream supplier. This represents the time required to receive and to ship orders. Therefore, on-hand inventory must be regulated with respect to the shipment delays.

Inventory managers make inventory replenishment decisions with local information at her/his echelon $i$ (e.g., the received orders from downstream customer, the on-hand inventory level, the placed orders to the upstream supplier, the shipments of upstream supplier, the shipments to downstream customer). S/he does not know the orders from the downstream customer that are currently being processed, on-hand inventory level of the upstream supplier, and the time at which $\mathrm{s} / \mathrm{he}$ receives the upstream supplier shipments. Her/his goal is to maximize profit or minimize the cost. From an optimization point of view, the overall considered cost function to minimize contains three components, namely the ordering cost, the inventory carrying cost and the shortage cost. Such costs are modeled accurately in Section 6.

Inventory managers must keep enough inventory to fulfill their customer needs and to avoid backlogs. To hold enough on-hand inventory, orders of the right quantity must be placed at the right time. Nevertheless, customer on-hand inventory depends on the supplier shipments and on-hand inventory level at the upstream suppliers. Variable time lags and impacts of previous decisions on the future ones add complexity to the continuous replenishment decision.

\section{Method}

Continuous replenishment decision-making has a dynamic and complex nature. This decision, including order quantity and order time, is characterized by time lags and impacts of previous decisions on future ones. The laboratory experiment is a suitable research tool to uncover the dynamic and complex nature of continuous replenishment decisions and address decision makers' cognitive limitations (e.g., Chatfield et al., 2004; Croson and Donohue, 2002). The laboratory experiment provides a stable and controlled environment, which eliminates possible noises and external perturbations (Katok, 2011). It provides the opportunity to observe decision maker's behavior over time that ensures multiple, repeatable, and reliable observations. To run the laboratory experiment, an appropriate platform is needed.

\subsection{Experimental platform}

We implement a role-playing platform developed by Montreuil et al. (2008). In this platform, continuous inventory replenishment is considered, which means that both order quantity and order time are variable. This is the major difference with respect to the well-known beer distribution game in which variable order quantities are placed within a fixed time interval between orders. More precisely, continuous review means here that the decision maker can 
always have access to the relevant identified information (e.g., on the inventory level s/he has to manage). Therefore, $\mathrm{s} /$ he can place an order anytime. This is opposed to periodic review, for which the opportunity (in contrast with the obligation) to place an order follows a fixed frequency (e.g., once a week).

The platform, which mimics the inventory replenishment dynamics in a decentralized sequential supply chain, consists of four echelons representing the roles of retailer, wholesaler, distributor, and factory. Market sends purchase orders to the retailer. The retailer delivers the requested items out of her/his on-hand inventory and if necessary, sends replenishment orders to the wholesaler. Likewise, wholesaler delivers the requested items out of her/his on-hand inventory, sends replenishment orders to and receives items from the distributor, who in turn sends replenishment orders to and receives items from the factory. The factory produces the items. There is no inventory capacity limitation. Figure 1 shows the structure supply chain in the experiment. Dotted (resp. solid) lines indicate orders (resp. shipments). Filled (resp. empty) rectangles represent echelons with human participants (resp. computer agents). Human participants, thereafter subjects, play the roles of the retailer, wholesaler and distributor, and computer agents play the factory and the market roles. None of the subjects are aware of the fact that the factory role is simulated by the computer. The market demand is stable. It follows a normal distribution with a mean (resp. standard deviation) of 3000 (resp. 500) units/day (truncated at zero). This daily demand is randomly split in two orders per day. In other words, this means that one can expect two orders of 1500 units every day (from the market). The demand information is unknown to the subjects.

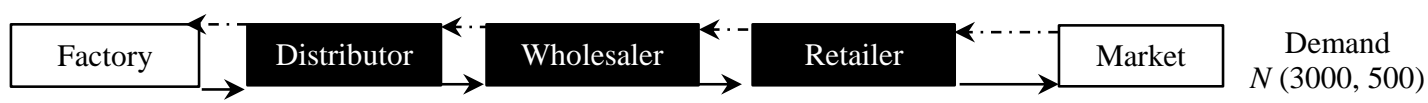

Figure 1: Structure of the supply chain used in the experiment

\subsection{Experimental procedure and experimental settings}

The experiment proceeds as follows: a subject enters the laboratory at an appointed time, and is randomly assigned to a team and a role. Before starting the experiment, we provide 4-5 pages handout explaining the instructions of the experiment and a summary of the role information to each subject. After that, the subjects read the instructions of the experiment, their questions are answered, and the experiment interface is then presented. Subjects are asked to play the simulation for almost 30 days ( 20 minutes) as a warm-up exercise to become familiar with the interface. After the warm-up session, we examine the subjects understanding of the experiment and their decision task in a short quiz to assure they acquire a sufficient knowledge. To avoid an end of the experiment behavior, the duration of the simulation is not announced in advance. The experiment lasts for 120 days (55 minutes) followed by a debriefing session. After the debriefing session, the subjects are asked to fill in a post-experiment questionnaire to reflect their replenishment policies during the experiment. The subjects are paid based on their performance during the experiment.

To examine the dynamics of inventory replenishment in a decentralized supply chain, the information availability is set as local (Lee and Billington, 1993): subjects are not allowed to communicate with anyone during the experiments and have visibility only on their own echelon dynamics. Subjects have a permanent access to the following information: the received order quantity and order time from downstream customer, their own on-hand inventory, the placed order quantity and order time with the upstream supplier, the shipments of upstream supplier, the shipments to downstream customer, and their performance measures. For all echelons, the 
holding (resp. backorder, fixed ordering) cost is $0.75 \$ /$ unit/day (resp. $1.5 \$ /$ unit/day, $15 \$$ ), the initial on-hand inventory is 9000 units, and the benefit is $2 \$ /$ item. The price of the beer at the factory (resp. distributor, wholesaler and retailer) is $4 \$$ item (resp. 6, 8 and 10).

\subsection{Subjects}

48 undergraduate and graduate students (with obvious knowledge in inventory management) from two Swiss universities (namely EPFL and UNIL), and engineering and business majors participate to the experiment for payoff contingent on their performance. The subjects consisted of $39 \%$ women and $61 \%$ men, with $24 \%$ of students in their bachelor, $44 \%$ in their master, and $22 \%$ in their $\mathrm{PhD}$. The experiment was conducted in the 2011 spring semester. The earning is calculated as in Equation (1). The subjects could earn $10 \mathrm{CHF}$ for showing up to the experiment, and up to $50 \mathrm{CHF}$ based on their performance. The maximum earning is approximately equivalent to two hours wages for a student job on campus.

$$
\text { Earning }=\operatorname{Min}\left\{50, \text { Show-up fee }(10 \mathrm{CHF})+40 \mathrm{CHF}^{*}\left(\frac{\text { Your profit }}{\text { Average of the role profit }}\right)\right\}
$$

\section{Statistical analyses and results}

The data set consists of 16 teams of supply chain. Observation of the experiment leads to the elimination of four supply chains from the data sample, as the related subjects did not make properly use of the experimental platform. Thus, we report on the results of $\mathrm{N}=12$ supply chains. Statistical analysis is carried out using SPSS 21 . Results of the experiments are analyzed on the individual and the echelon levels. The individual level analysis refers to the obtained data for each subject, whereas the echelon level analysis refers to the aggregation of individual results for a considered role. Unexpectedly, it will be observed that the cost can be strongly affected by upstream variabilities. Indeed, the literature usually looks downstream for the source of variability and fluctuations.

\subsection{Inventory replenishment}

\subsubsection{Mean replenishment decision indicators}

Table 1 summarizes the mean of order quantity and order time intervals for all individuals and echelons. For all the teams, the replenishment decision indicators increase while moving from retailer to wholesaler. However, only $58 \%$ of the distributors increase their replenishment decision indicators compared to wholesalers.

Table 1. Mean order quantity $(O Q)$ and order time intervals (OT, in minutes)

\begin{tabular}{lllll}
\hline Team & Distributor & Wholesaler & Retailer & Market \\
\hline
\end{tabular}




\begin{tabular}{ccccccccc}
\hline & $O Q$ & $O T$ & $O Q$ & $O T$ & $O Q$ & $O T$ & $O Q$ & $O T$ \\
\hline 1 & 7348 & 61 & 3137 & 25 & 1022 & 8 & 1508 & 12 \\
2 & 7875 & 56 & 6911 & 48 & 5433 & 43 & 1508 & 12 \\
3 & 4890 & 40 & 7140 & 59 & 1872 & 15 & 1508 & 12 \\
4 & 3529 & 29 & 3802 & 31 & 3381 & 27 & 1508 & 12 \\
5 & 7039 & 58 & 2813 & 22 & 1798 & 15 & 1508 & 12 \\
6 & 9711 & 77 & 4751 & 38 & 3781 & 30 & 1508 & 12 \\
7 & 5786 & 47 & 7957 & 65 & 5530 & 45 & 1508 & 12 \\
8 & 6566 & 56 & 5839 & 48 & 5304 & 43 & 1508 & 12 \\
9 & 3733 & 31 & 6992 & 57 & 2627 & 21 & 1508 & 12 \\
10 & 1000 & 8 & 4000 & 31 & 3512 & 27 & 1508 & 12 \\
11 & 3415 & 28 & 2645 & 21 & 1003 & 8 & 1508 & 12 \\
12 & 9634 & 73 & 3236 & 24 & 1000 & 8 & 1508 & 12 \\
\hline Mean of each echelon & 5877 & 47 & 4935 & 39 & 3022 & 24 & 1508 & 12 \\
\hline
\end{tabular}

Figure 2 displays the minimum, maximum and mean of the replenishment decision indicators for each role over the game. The mean of order quantity and order time intervals are $(1508,12)$, $(3022,24),(4935,39),(5877,47)$ for market $(M)$, retailer $(\mathrm{R})$, wholesaler $(\mathrm{W})$ and distributor (D), respectively. Table 2 shows the SRCC (i.e., the Spearman Rank Correlation Coefficient, (Spearman, 1904)) among the replenishment decision indicators of each echelon. Note that $* * *$ and $* *$ are significant at $\mathrm{p}<0.01$ and 0.05 levels, respectively (this also holds for Tables $6-9$ ). Because the current datasets are not normally distributed, using a nonparametric test as SRCC is important in data analysis. We remark a strong positive correlation between mean order quantity and mean order time interval for each echelon. Results show that wholesaler's replenishment decision indicators are positively correlated with the immediate customer, but distributor's replenishment decision indicators are not.
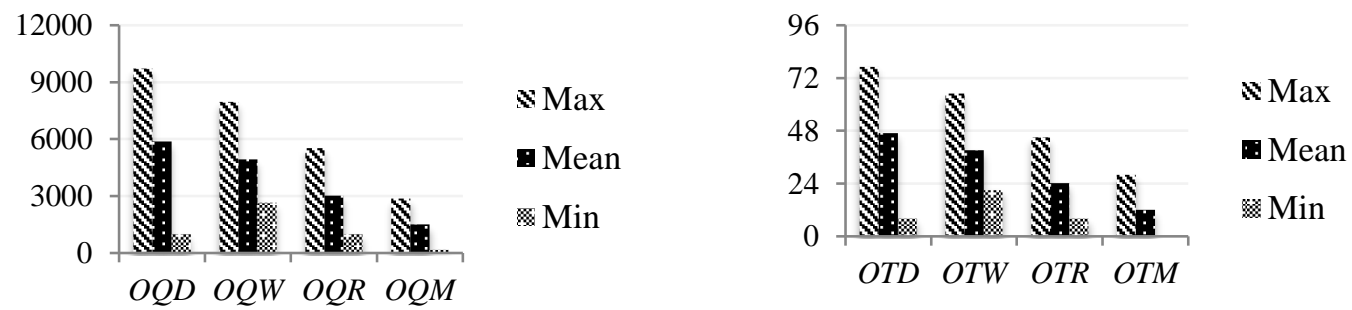

Figure 2. (a) Order quantity (in items) and (b) order time interval (in hours) for each echelon

Table 2. SRCC matrix among replenishment decision indicators of each echelon

\begin{tabular}{ccccccc}
\hline & $O Q^{D}$ & $O Q^{W}$ & $O Q^{R}$ & $O T^{D}$ & $O T^{W}$ & $O T^{R}$ \\
\hline$O Q^{D}$ & 1 & & & & & \\
$O Q^{W}$ & 0 & 1 & & & & \\
$O Q^{R}$ & 0.035 & $0.699^{* *}$ & 1 & & & \\
$O T^{D}$ & $0.970^{* * *}$ & -0.095 & -0.079 & 1 & & \\
$O T^{W}$ & -0.018 & $0.989^{* * *}$ & $0.709^{* *}$ & -0.095 & 1 & \\
$O T^{R}$ & 0.057 & $0.676^{* *}$ & $0.988^{* * *}$ & -0.046 & $0.679^{* *}$ & 1 \\
\hline
\end{tabular}

The analysis of the results of this subsection 5.1.1 leads to two main observations. 
Observation 1: The mean order quantity and mean order time intervals tend to increase while moving upstream along the supply chain under continuous review.

Observation 2: Retailer and wholesaler replenishment decision indicators are positively correlated, whereas distributor replenishment indicators have no significant correlation with its immediate customer.

\subsubsection{Variability of replenishment decision indicators}

The coefficient of variation $(\mathrm{CV})$ is used to study the variability of the replenishment decision indicators. $C V$ is a relative variation measure and is a way to interpret the relative magnitude of the standard deviation by dividing it by the mean. Table 3 provides an overview of the replenishment decision indicators variability. We classify the variability as follows: (1) Low variability: $0 \leq C V_{O Q}<0.5$ and $0 \leq C V_{O T}<0.5$; (2) Medium variability: $0.5 \leq C V_{O Q}<1$, and/or $0.5 \leq C V_{O T}<1$; High variability: $C V_{O Q} \geq 1$, and/or $C V_{O T} \geq 1$. Table 3 shows that: (1) $6 \%$ of the subjects ( 2 out of 36 ) have low variability for both order quantity and order time intervals; (2) $53 \%$ of the subjects (19 out of 36) have medium variability for at least one of the two replenishment indicators; (3) $41 \%$ of the subjects (15 out of 36) have high variability for both replenishment indicators. Furthermore, among all the subjects, one retailer (R12), one wholesaler (W10), and one distributor (D10) have fixed order quantity $\left(C V_{O Q}=0\right)$ and variable order time intervals policy. All the other subjects (91.5\%) follow a variable order quantity and variable order time intervals policy.

Table 3. Variability on order quantity and order time intervals for each individual

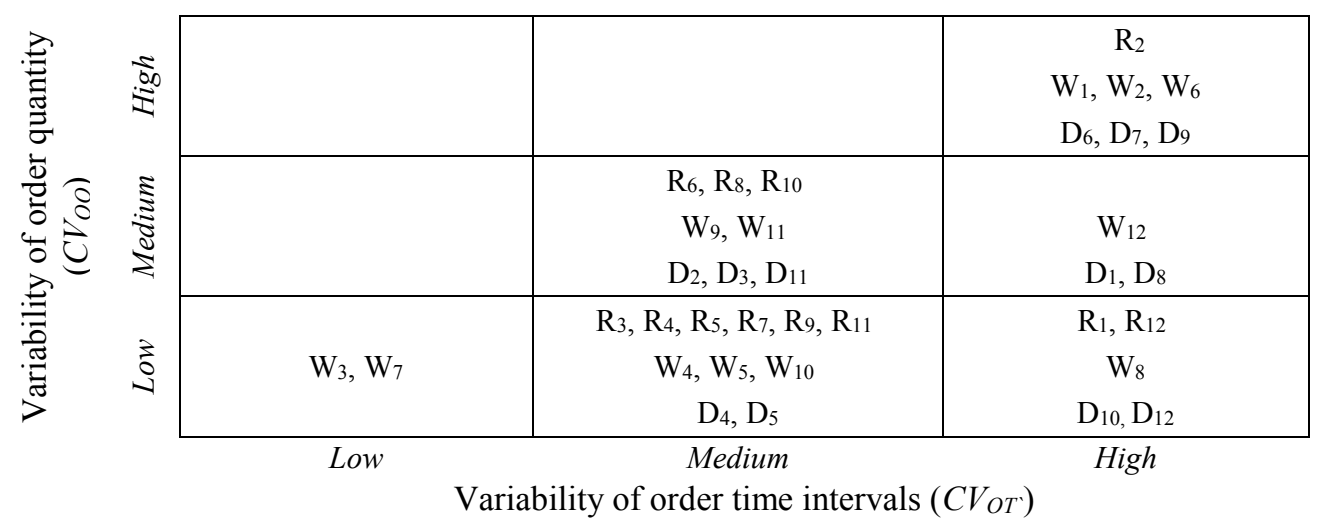

Table 4 summarizes the numbers of individuals for each variability level. The low variability results show that only $17 \%$ wholesalers place orders with low variability, where no retailer or distributor belongs to that variability range. The tendency of the subjects with medium variability decreases from retailers $(67 \%)$, wholesalers $(33 \%)$, to distributors $(25 \%)$. For the high variability, the variability of the replenishment decision indicators tends to be higher on the upstream echelons. Therefore, subjects in upstream echelons place more variable order quantities at more variable time intervals. There is no team with low variability condition. There are only 2 teams out of 12 , with all three players, showing medium variability of their replenishment decision indicators. Table 4 also shows that there is at least one subject in a team, dominantly among the upstream echelons, who introduces the medium or high variability on her/his inventory replenishment decision.

Table 4. Number of individuals on low, medium, and high variability conditions 


\begin{tabular}{|c|c|c|c|c|c|}
\hline $\begin{array}{c}\text { Variability } \\
\text { level }\end{array}$ & $D$ & $W$ & $R$ & $\begin{array}{c}\text { Team \# with this } \\
\text { condition }\end{array}$ & $\begin{array}{c}\text { Team \# with 2 echelons following this } \\
\text { condition }\end{array}$ \\
\hline Low & 0 & $17 \%$ & 0 & - & - \\
\hline Medium & $25 \%$ & $33 \%$ & $67 \%$ & 4,5 & 12 \\
\hline High & $75 \%$ & $50 \%$ & $33 \%$ & - & $6,11,12$ \\
\hline
\end{tabular}

Figure 3 illustrates the variability of the replenishment decision indicators for each echelon. The aggregated results at the echelon level show that retailers place orders with higher variability of the order quantity and order time interval than the market demand $\left(C V_{O Q} Q^{R}=0.43\right.$, $\left.C V_{O T}{ }^{R}=0.89\right)$. Wholesaler receives these fluctuating orders from retailer and increases the variability of order quantity, while the variability of order time intervals stays almost unchanged $\left(C V_{O Q}{ }^{W}=0.62, C V_{O T}{ }^{W}=0.88\right)$. Finally, the distributor increases the variability of both order quantity and order time $\left(C V_{O Q}{ }^{D}=0.69, C V_{O T}{ }^{D}=1.27\right)$. Thus, there is an increase of the variability of order quantity and order time interval while moving upstream from market along the supply chain.

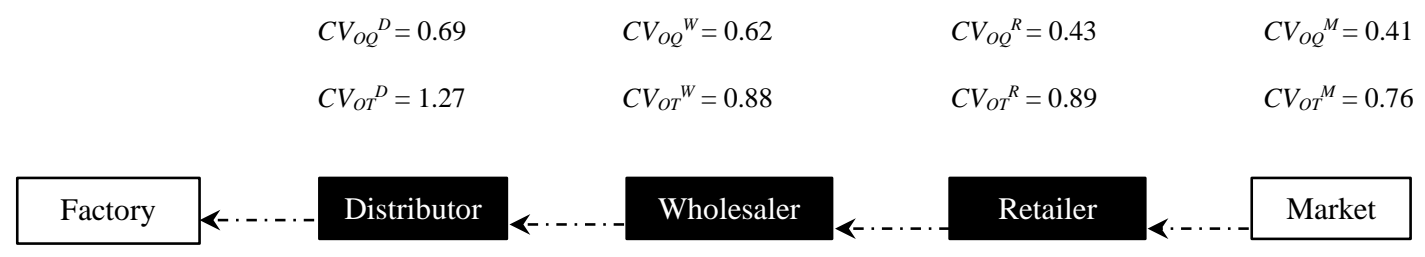

Figure 3: Variability of order quantity and order time intervals at the echelon level

Under continuous review, the results of this subsection leads to two main observations.

Observation 3: The relative number of subjects with high variability of their replenishment decision indicators tends to increase while moving upstream from market along the supply chain.

Observation 4: The variability of replenishment decision indicators for each echelon tends to increase while moving upstream from market along the supply chain.

\subsection{Costs}

Table 5 shows the mean of backorder cost $(B C)$, holding cost (HC), and total cost (TTC) for each echelon. The cost components that a subject incurred over the experiment in the participatory simulation platform consists of backorder cost, holding cost, and purchasing cost. Because purchasing cost is highly correlated with order quantity and order time intervals of each player, we exclude it from our analysis and mainly focused on backorder and holding cost. Thus, the total cost is their sum. The unit cost components are similar across the echelons (see Section 4.2). Results show that $T T C$ increase from retailer to distributor. The $H C$ column shows an increase from retailer to distributor, and the $B C$ column shows wholesaler incurs less backorder cost compared to retailer and distributor.

Table 5. Mean of backorder (BC), holding cost (HC), and total cost (TTC) 


\begin{tabular}{cccc}
\hline & $B C$ & $H C$ & TTC \\
\hline Retailer & 503,991 & 217,019 & 721,009 \\
Wholesaler & 497,937 & 486,766 & 984,703 \\
Distributor & 574,494 & 687,763 & $1,262,197$ \\
\hline
\end{tabular}

To investigate the relationship between the replenishment decision indicators with incurred costs, we perform Spearman rank correlation. Table 6 displays the link between replenishment decision indicators, backorder cost and holding cost. Results show that the backorder cost of distributor is positively correlated with its order quantity. Her/his holding cost is also positively correlated with the order quantity as well as with the order time intervals. Moreover, the same positive correlation is observed between wholesaler's backorder cost and both distributor's order quantity and order time intervals. No significant correlation is identified for any of the retailer's cost, nor for the wholesaler's holding cost.

Table 6. SRCC between replenishment decision indicators, holding and backorder costs

\begin{tabular}{ccccccc}
\hline & $B c^{D}$ & $H c^{D}$ & $B c^{W}$ & $H c^{W}$ & $B c^{R}$ & $H c^{R}$ \\
\hline$O Q^{D}$ & $0.538^{* *}$ & $0.587^{* * *}$ & $0.706^{* * *}$ & 0.182 & 0.357 & 0.531 \\
$O Q^{W}$ & 0.133 & 0.308 & 0.154 & 441 & -0.336 & 0.175 \\
$O Q^{R}$ & 0.028 & 0.091 & 0.339 & 0.385 & -0.168 & 0.147 \\
$O T^{D}$ & 0.417 & $0.644^{* * *}$ & $0.623^{* * *}$ & 0.053 & 0.406 & 0.41 \\
$O T^{W}$ & 0.112 & 0.295 & 0.088 & 0.442 & -0.372 & 0.123 \\
$O T^{R}$ & 0.007 & 0.117 & 0.411 & 0.315 & -0.159 & 0.209 \\
\hline
\end{tabular}

Table 7 reports the $S R C C$ between replenishment decision indicators and total costs (TTC) of each echelon. Results show a strong positive correlation between distributor TTC, distributor's order quantity, and order time intervals. Similarly, the retailer TTC is positively correlated with the distributor's order quantity and order time interval. TTC of wholesaler is also positively correlated with wholesaler's order quantity, but no significant correlation is found with the wholesaler's order time intervals.

Table 7. SRCC between replenishment decision indicators and total costs

\begin{tabular}{cccc}
\hline & $T T C^{D}$ & $T T C^{W}$ & $T T C^{R}$ \\
\hline$O Q^{D}$ & $0.727^{* * *}$ & $0.608^{* *}$ & $0.580^{* *}$ \\
$O Q^{W}$ & 0.364 & 0.336 & -0.301 \\
$O Q^{R}$ & 0.168 & 0.357 & -0.105 \\
$O T^{D}$ & $0.658^{* *}$ & 0.49 & $0.592^{* *}$ \\
$O T^{W}$ & 0.34 & 0.27 & -0.358 \\
$O T^{R}$ & 0.156 & 0.336 & -0.071 \\
\hline
\end{tabular}

Table 8 indicates the $S R C C$ between the variability of the replenishment decision indicators, and holding and backorder costs. Results show that the distributor backorder cost is positively correlated with the variability of the distributor's order quantity, wholesaler's order quantity, and order time intervals. The distributor holding cost appears to be significantly positively correlated with variability of the distributor's order quantity. No significant correlation is found for wholesaler backorder cost, retailer's backorder, and holding costs. 
Table 8. SRCC between the variability on replenishment decision indicators, holding and backorder costs

\begin{tabular}{lcccccc}
\hline & $B c^{D}$ & $H c^{D}$ & $B c^{W}$ & $H c^{W}$ & $B c^{R}$ & $H c^{R}$ \\
\hline$C V_{\text {OQD }}$ & $0.601^{* *}$ & $0.678^{* *}$ & 0.315 & 0.469 & -0.196 & 0.343 \\
$C V_{\text {OQW }}$ & $0.860^{* * *}$ & 0.483 & 0.371 & $0.503^{*}$ & 0.014 & 0.476 \\
$C V_{\text {OQR }}$ & 0.091 & -0.021 & 0.413 & $0.636^{* *}$ & 0.224 & -0.021 \\
$C V_{\text {OTD }}$ & 0.196 & 0.406 & 0.497 & 0.315 & 0.175 & 0.021 \\
$C V_{\text {OTW }}$ & $0.613^{* *}$ & 0.165 & 0.305 & 0.2 & 0.242 & 0.406 \\
$C V_{\text {OTR }}$ & 0.329 & -0.333 & 0.098 & 0.238 & -0.137 & 0.378 \\
\hline
\end{tabular}

Table 9 depicts the $S R C C$ among variability of the replenishment decision indicators and TTC. We observe: (1) a strong correlation between the distributor's TTC, and the wholesaler's and distributor's variability of order quantity; (2) a positive correlation between wholesaler's and retailer's variability of order quantity and their supplier TTC (distributor and wholesaler, respectively); (3) no significant correlation between the variability of order time intervals and TTC of any echelon.

Table 9. SRCC between the variability of replenishment decision indicators and total costs

\begin{tabular}{lccc}
\hline & $T T C^{D}$ & $T T C^{W}$ & $T T C^{R}$ \\
\hline$C V_{\text {OQD }}$ & $0.846^{* * *}$ & 0.28 & -0.20 \\
$C V_{\text {OQW }}$ & $0.867^{* * *}$ & 0.427 & 1 \\
$C V_{\text {OQR }}$ & 0.119 & $0.580^{* *}$ & 0.193 \\
$C V_{\text {OTD }}$ & 0.371 & 0.545 & 0.217 \\
$C V_{\text {OTW }}$ & 0.452 & 0.375 & 0.147 \\
CV $V_{\text {OTR }}$ & 0.063 & 0.494 & 0.392 \\
& & & -0.032 \\
\hline
\end{tabular}

\section{Normative model}

This section presents a simplified normative model for inventory replenishment in a decentralized supply chain. The goal is to identify the optimal replenishment indicators that lead to minimize total cost in a decision situation as close as possible to the experiment. The model is based on three assumptions: (AI) Profit is considered to be essentially dependent on cost rather than income so that the model can be simplified to a cost optimization. (AII) Order quantity $O Q$ and order time interval $O T$ are constant (note that $O T$ can be very small, which makes the link with the continuous review replenishment policy). This assumption considers a stable replenishment decision behavior to be compared with the mean order quantity and mean order time intervals indicated in Section 6.1. (AIII) Replenishment order time interval $O T^{i}$ is larger than received time interval $O T^{i-1}$. This assumption is considered to simplify the optimization process by restricting the solution space to that observed in the experiment.

Figure 4 provides an illustration of the modeling situation within a single replenishment cycle (hereafter cycle) at echelon $i$. As defined earlier, $i$ is the echelon index ( $i=[R, W, D] ; R$ : retailer, $W$ : wholesaler, $D$ : distributor) in a supply chain, and $i=M$ for the market index. 


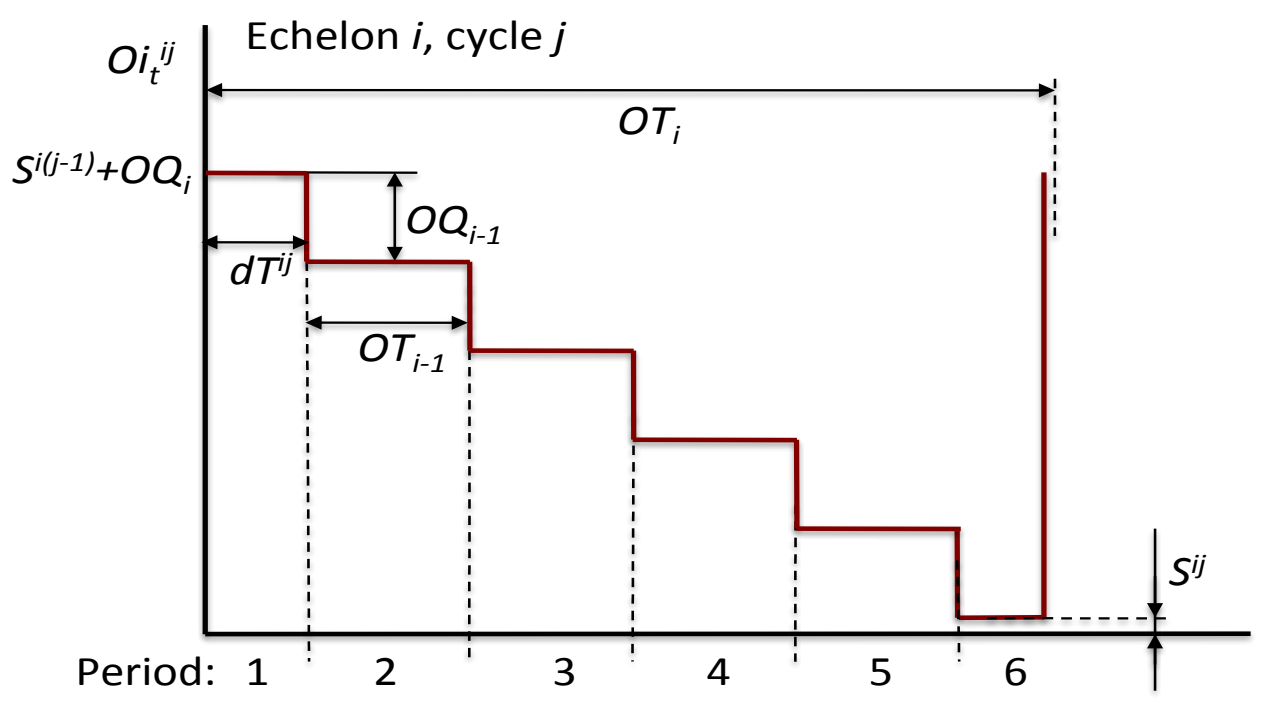

Figure 4. Modeling characteristics for a single replenishment cycle at echelon $i$

\subsection{Definitions and notation}

The cycle length is given by the replenishment order time interval $O T^{i}$. Definitions and notation used in the model are defined as below. The six first elements are part of the data.

- $D^{i}$ : annual demand at echelon $i ; D^{l}=$ market demand

- $\quad r^{i}$ : inventory carrying cost rate in \% per unit per period at echelon $i\left(r^{i} \geq r^{i+1}\right)$

- $\quad v^{i}$ : value of a single unit in inventory at echelon $i$

- $B^{i}$ : backorder cost per unit per period at echelon $i$

- $F^{i}$ : fixed order cost at echelon $i$

- $O I_{0}^{i}$ : initial on-hand inventory at time 0

- $O Q^{i}$ : mean order quantity placed to supplier $i+1$

- $O T^{i}$ : mean time interval between two consecutive orders placed to supplier $i+1$

For cycle $\mathrm{j}$ (with $\mathrm{j}>0$ ), where a cycle is defined as max $\left(O T^{i}, O T^{i-1}\right)$, we define:

- $O I_{t}^{i j}$ : inventory at period $t$ within cycle $j$ at echelon $i$

- $I C_{t}^{i j}$ : inventory carrying cost for period $t$ within cycle $j$ at echelon $i$

- $S^{i j}$ : inventory level at the end of cycle $j$ (which can be negative) at echelon $i$

- $d T^{i j}$ : time between the beginning of cycle $j$ and the first inventory level change of cycle $j$ at echelon $i$

- $I C C^{i j}$ : inventory carrying cost per cycle $j$ at echelon $i$

- $O C C^{i j}$ : ordering cost per cycle $j$ at echelon $i$

- $S C C^{i j}$ : backorder cost per cycle $j$ at echelon $i$

- $\quad N^{i c}$ : number of full replenishment cycles per year at echelon $i$

Let $N C^{i}$ be a quantity defined as $D^{i} / O Q^{i}$. The number of full replenishment cycles per year is then the truncated value of $N C^{i}$, and the annual cycle cost is given in Equation (2).

$$
A C^{i}=\left[\frac{N C^{i}}{N^{i c}}\right] \times \sum_{j=1}^{N^{i c}}\left(I C C^{i j}+O C C^{i j}+S C C^{i j}\right)
$$


Let $\tau^{j}$ be the truncated value of $\left(\mathrm{OT}^{\mathrm{i}}-\mathrm{dT}^{\mathrm{ij}}\right) / O T^{i-1}$. In Figure 4, we compute $\tau^{j}$ as follows: $(5.3-0.5) / 1$, which is then truncated at 4 . The value $\tau^{j}$ corresponds to the number of full periods with duration $O T^{i-1}$. Three types of time period have to be considered: (1) $t=1$ : the first time period of duration $d T^{i j}$ is likely to be smaller than $O T^{i-1}$; (2) $2 \leq t \leq \tau^{j}+1$ : each of the intermediate time periods is equal to $O T^{i-1}$; (3) $t=\tau^{j}+2$ : period is again likely to be smaller than $O T^{i-1}$.

\subsection{Optimization problem statement and cost computation}

For echelon $i$, we have to optimize $O Q^{i}$ and $O T^{i}$ in order to minimize the annual costs $A C^{i}$, which consists of three components: inventory carrying costs $I C^{i}$, ordering cost $O C^{i}$, and shortage costs $S C^{i}$. The three cost components $I C C^{i j}, O C C^{i j}$ and $S C C^{i j}$ are first computed and later cumulated according to Equation (2) to obtain the total cost per year $A C^{i}$. The inventory carrying cost per cycle is given by Equation (3).

$$
\begin{aligned}
& I C C^{i j}=r^{i} v^{j}\left\{\begin{array}{l}
\max \left(0, O I_{t=1}^{i j}\right) \times\left[d T^{i j}\right]+\sum_{t=2}^{\tau^{j}+1} \max \left(0, O I_{t}^{i j}\right) \times\left[O T^{i-1}\right] \\
+\max \left(0, O I_{\tau^{j}+2}^{i j}\right) \times\left[O T^{i}-\tau^{j} O T^{i-1}-d T^{i j}\right]
\end{array}\right\} \\
& O I_{t}^{i j}=O Q+S^{(j-1)}-(t-1) O Q^{i-1} \\
& d T^{i(j+1)}=\left\lfloor O T^{i-1}-\left(O T^{i}-\tau^{j} O T^{i-1}-d T^{i j}\right)\right\rfloor
\end{aligned}
$$

The remaining inventory at the end of cycle $j$ is $S^{i j}=O I_{\tau^{j}+2}^{i j}$. The initial conditions are: $d T^{i(j=1)}=\frac{1}{2} O T^{i-1}$, and $S^{i(j=0)}$ is equal to a given value. The ordering cost per cycle is $O C C^{i j}=F^{i}$. The backorder cost per cycle is given in Equation (6).

$$
S C C^{i j}=B^{i}\left\{\begin{array}{l}
\max \left(0,-O I_{t=1}^{i j}\right) \times\left[d T^{i j}\right]+\sum_{t=2}^{\tau^{j}+1} \max \left(0,-O I_{t}^{i j}\right) \times\left[O T^{i-1}\right] \\
+\max \left(0,-O I_{\tau^{j}+2}^{i j}\right) \times\left[O T^{i}-\tau^{j} O T^{i-1}-d T^{i j}\right]
\end{array}\right\}
$$

\subsection{Optimization heuristics}

As the demand rate is constant (fixed $O Q$ and $O T$ ), assumption (AII) implies $O Q^{i} \geq O Q^{i-1}$. Also, as the case of a decentralized supply chain is considered, a single model identical for all echelons is sufficient. To simplify the notation, the subscript $i$ will therefore not be used in this subsection. The model and the optimization procedure are used to answer to the following questions: (Q1) What is the optimal OT for a given OQ? (Q2) What are the optimal OT and $O Q$ when both are variable? The optimization procedures for these two questions are described below.

Procedure $O P T I M(O T \mid O Q)$ aims at finding the $O T$ value which minimizes the annual costs if $O Q$ is imposed. Starting from a very small value of $O T$, its main idea is to evaluate the most relevant and promising $O T$ values, with small steps of $\Delta T=1 / 100$ day $\approx 14$ minutes. Smaller 
steps are not relevant from a practical standpoint. Indeed, one can reasonably assume that a decision maker will not reconsider placing an order more often than every 15 minutes.

Procedure $O P T I M(O Q)$ aims at finding the $O Q$ value which minimizes the annual costs. Starting from the smallest reasonable value, its main idea is to evaluate the most relevant and promising $O Q$ values (with small steps of $\triangle Q=100$ units), for which the best associated $O T$ values can be computed with Procedure $\operatorname{OPTIM}(O T \mid O Q)$. Again, smaller steps for $\triangle Q$ are not relevant, as one can reasonably assume that the smallest possible quantity to order cannot be below a threshold of 100 units.

The two above procedures were implemented in $\mathrm{C}$ and their computing time is negligible. As it is always possible to use smaller $\Delta Q$ and $\Delta T$ increments (although totally unrealistic from a practical standpoint), we cannot formally assume that the provided solutions are mathematically optimal. But as the considered increments $\Delta Q$ and $\Delta T$ have the smallest possible realistic values, the two above procedures are likely to find optimal solutions from a managerial point of view, and within a faction of second. Note that additional experiments were performed with smaller unrealistic increments, but without reducing the costs by more than $0.1 \%$. Because of such an efficiency, there is no need to investigate for more refined or quicker optimization approaches.

\section{Procedure $O P T I M(O T \mid O Q)$ for a given value of $O Q$ (for question $(Q 1)$ )}

Let $A C(O T \mid O Q)$ be the annual cost function assuming $O Q$ is given, the values of $O Q$ being those observed in the experiment (see Table 1). A time increment (between two orders) of 0.01 day ( $\approx 14$ minutes) is chosen. This value corresponds to a real time interval $\approx 0.3$ second for the subjects and is therefore considered to be small enough.

Initialization

- $\quad$ set $O T=15$ minutes (smallest reasonable time interval between 2 orders)

- choose a value for $\Delta \mathrm{T}$ (time increment between 2 orders, e.g. 1/100 day)

- compute $A C(O T \mid O Q)$

- $\quad$ set $O T^{*}=O T$ and $F^{*}=A C(O T \mid O Q)$ (storage of the best OT and cost values)

While $A C(O T \mid O Q)<2 F^{*}$, do: (i.e., while the annual cost is not above a reasonable threshold)

- $\quad$ set $O T=O T+\triangle T$

- compute $A C(O T \mid O Q)$

- if $A C(O T \mid O Q)<F^{*}$, set $O T^{*}=O T$ and $F^{*}=A C\left(O T^{*} \mid O Q\right)$

Output: $O T^{*}$ and $F^{*}=A C\left(O T^{*} \mid O Q\right)$, respectively denoted $O T^{*}(O Q)$ and $A C\left[O T^{*}(O Q)\right]$.

Procedure $O P T I M(O Q)$ (for question $(Q 2)$ )

Initialization 
- $\quad$ set $O Q=O Q^{(i-1)}$ (we start with the smallest reasonable order quantity)

- choose a value for $\triangle Q$ (quantity increment of an order, e.g. 100 units)

- perform $O P T I M(O T \mid O Q)$ which returns $O T^{*}(O Q)$ and $A C\left[O T^{*}(O Q)\right]$

- $\operatorname{set} O Q^{*}=O Q, O T^{*}=O T^{*}(O Q), F^{*}=A C\left[O T^{*}(O Q)\right]$ (store the best decisions and cost)

While $A C[O T(O Q)]<2 F^{*}$, do: (i.e., while the annual cost is not above a reasonable threshold)

- $\quad$ set $O Q=O Q+\triangle Q$

- $\quad$ perform $O P T I M(O T \mid O Q)$ to compute $O T^{*}(O Q)$

- if $A C\left[O T^{*}(O Q)\right]<F^{*}$, set $O Q^{*}=O Q, O T^{*}=O T^{*}(O Q)$ and $F^{*}=A C\left[O T^{*}(O Q)\right]$

Output: $O Q^{*}, O T^{*}$ and $A C\left[O T^{*}, O Q^{*}\right]$.

\subsection{Optimization results}

As for the experiment (see Subsection 4.2), for all echelons, the holding (resp. backorder, fixed ordering) cost is $0.75 \$$ unit/day (resp. $1.5 \$ /$ unit/day, $15 \$$ ) and the initial on-hand inventory is 9000 units.

The $O P T I M(O T \mid O Q)$ procedure is first applied to find the optimum time $O T^{*}$ between orders for order quantities equal to the average values observed in the experiment $O Q$ values in Table 1. The results are indicated in Table 10, together with those of the experiment. As it can be observed, these two sets of values $\left(O T^{*}\right.$ and $\left.O T\right)$ are very close, indicating that the subjects apply an ordering periodicity, which is well adjusted to the order quantity. However, the computed time interval $O T^{*}$ are systematically, even if slightly (approx. 2\%), larger than the experimental values $O T$. These larger $O T^{*}$ values lead to an increase of the backorder cost, but to a very significant decrease of the inventory carrying cost and total cost (25\% to 55\%).

Table 10. Computed results obtained using the OPTIM $(O T \mid O Q)$ procedure for $O Q$ values equal to experimental average order quantities

\begin{tabular}{|l|c|c|c|c|}
\hline & Distributor & Wholesaler & Retailer & Market \\
\hline Experimental $O Q(=$ average in Table 1) & 5877 & 4935 & 3022 & 1508 \\
\hline Experimental $O T(=$ average in Table 1) & 47 & 39 & 24 & 12 \\
\hline Computed order time interval $O T^{*}$ & 47.48 & 39.96 & 24.48 & 12 \\
\hline $\begin{array}{l}\text { Inventory carrying cost (\$) based on } \\
\text { experimental order parameters }(O Q, O T)\end{array}$ & $3,513,302$ & $4,418,792$ & $3,657,628$ & \\
\hline $\begin{array}{l}\text { Inventory carrying cost (\$) based on } \\
\text { computed order parameters }\left(O Q, O T^{*}\right)\end{array}$ & $2,266,992$ & $1,918,365$ & $1,569,683$ & \\
\hline $\begin{array}{l}\text { Shortage cost (\$) based on experimental order } \\
\text { parameters }(O Q, O T)\end{array}$ & 4,205 & 0 & 0 & \\
\hline $\begin{array}{l}\text { Shortage cost (\$) based on computed order } \\
\text { parameters }\left(O Q, O T^{*}\right)\end{array}$ & 385,776 & 141,230 & 82,867 & \\
\hline $\begin{array}{l}\text { Total cost (\$) based on experimental order } \\
\text { parameters }(O Q, O T)\end{array}$ & $3,519,190$ & $4,420,798$ & $3,660,904$ & \\
\hline $\begin{array}{l}\text { Total cost (\$) based on computed order } \\
\text { parameters }\left(O Q, O T^{*}\right)\end{array}$ & $2,654,452$ & $2,061,600$ & $1,655,826$ & \\
\hline
\end{tabular}

The results for $O Q^{*}$ and $O T^{*}$ obtained with $\operatorname{OPTIM}(Q)$ are reported in Table 11, together with the corresponding average experimental values $O Q$ and $O T$. Recall that the computed $O Q^{*}$ and $O T^{*}$ values of Table 11 are those leading to the minimum total cost under assumption (AII). 
With this constraint, it can be observed that the order policy used by the subjects in the experiment are not optimal, as order quantities and order time intervals are almost half of the computed values. The lower $O Q$ and $O T$ values lead to negligible backorder costs, but to a very significant increase of the inventory carrying costs and consequently of total costs $(147 \%$ to $253 \%$ ). This observation is discussed in a more detailed way in the next section.

Table 11. Computed results obtained using the OPTIM(OQ) procedure

\begin{tabular}{|l|c|c|c|c|}
\hline & Distributor & Wholesaler & Retailer & Market \\
\hline Experimental $O Q(=$ average in Table 1$)$ & 5877 & 4935 & 3022 & 1508 \\
\hline Computed order quantity $O Q^{*}$ & 9635 & 8122 & 5708 & 1508 \\
\hline Experimental $O T(=$ average in Table 1$)$ & 47 & 39 & 24 & 12 \\
\hline Computed order time interval, $O T^{*}$ & 77.44 & 66.16 & 46.44 & 12 \\
\hline $\begin{array}{l}\text { Inventory carrying cost }(\$) \text { based on } \\
\text { experimental order parameters }(O Q, O T)\end{array}$ & $3,513,302$ & $4,418,792$ & $3,657,628$ & \\
\hline $\begin{array}{l}\text { Inventory carrying cost }(\$) \text { based on } \\
\text { computed order parameters }\left(O Q^{*}, O T^{*}\right)\end{array}$ & $1,877,624$ & $1,555,181$ & $1,257,592$ & \\
\hline $\begin{array}{l}\text { Shortage cost }(\$) \text { based on experimental } \\
\text { order parameters }(O Q, O T)\end{array}$ & 4,205 & 0 & 0 & \\
\hline $\begin{array}{l}\text { Shortage cost }(\$) \text { based on computed order } \\
\text { parameters }\left(O Q^{*}, O T^{*}\right)\end{array}$ & 513,702 & 372,923 & 188,576 & \\
\hline $\begin{array}{l}\text { Total cost }(\$) \text { based on experimental order } \\
\text { parameters }(O Q, O T)\end{array}$ & $3,519,190$ & $4,420,798$ & $3,660,904$ & \\
\hline $\begin{array}{l}\text { Total cost }(\$) ; \text { based on computed order } \\
\text { parameters }\left(O Q^{*}, O T^{*}\right)\end{array}$ & $2,392,364$ & $1,929,323$ & $1,447,902$ & \\
\hline
\end{tabular}

\section{Discussion}

Among the limitations of this study, one could first mention that the students' experiments are bounded by the lack of tacit knowledge, and do not accurately represent true decision-making and risk taking in the real world, whereas the normative model was designed with expert knowledge. Second, even if the considered sample size (i.e., $\mathrm{N}=12$ ) could be pointed out as being rather small, it allows designing a first set of interesting conclusions. Consolidating these conclusions with a larger team of experts, and with various supply chain structures and demand patterns, is left as an avenue of research resulting from - and motivated by - this first study.

Previous studies of the ordering behavior in decentralized supply chains focus on order quantity only. This work allowing both order time and order quantity to vary constitutes an extension and allows an interesting comparison. The first significant conclusion is that in both cases (with or without time related decision), the order quantity $O Q$ used by subject increases while moving upstream along the supply chain. The second meaningful observation is that, when the time interval $O T$ is part of the replenishment decision, it increases similarly to the order quantity in case of a constant demand rate.

Order amplification is caused by both structural and behavioral reasons. Demand signal processing, order batching, price variation, and inventory rationing are structured characteristics that cause demand amplification (Lee et al., 1997). Decision maker bounded rationality to account for time lags and previous decisions feedbacks (Sterman, 1989), and uncertainty of individual decisions for the other supply chain members (Croson et al., 2012) are behavioral characteristics that amplify order. In this study, unknown market demand leads the subjects to uncertain forecasts that cause demand signal processing. Along with this structural 
reason, both above-mentioned behavioral causes play a role in the order amplification at each level of supply chain as one moves upstream from the market to distributor.

The results of the normative model show that the time intervals $O T$ used by subjects are almost optimally adapted to their order quantities $O Q$. Therefore, subjects adjust order quantity and time interval in order to obtain, on average, a supply rate close to the average demand rate. Note that the objective function $A C(O T \mid O Q)$ is convex in $O T$. However, comparing the experimental results to the normative model (Table 10) shows that the chosen $O T$ of the subject policy is not optimally adjusted to $O Q$. Indeed, the normative model indicates that a slightly longer (around 1\%) time interval OT leads to higher shortage costs, but much lower inventory carrying and total costs. This reveals that the subject risk reluctance is detrimental as it avoids reaching the optimal $O T$ for a given $O Q$.

Furthermore, the results obtained from the normative model indicate that subjects do not use the best possible combination of $(O Q, O T)$ as the best values found by the optimization heuristics $\left(O Q^{*}, O T^{*}\right)$ are roughly twice the experimental ones. It must be noticed here that the objective function $A C(O T, O Q)$ is not convex in $O Q$. The subject policy, with lower $O Q$ and $O T$, leads to lower backorder costs, but much higher inventory carrying cost and consequently for total costs (147\% to $253 \%)$. The use of order time intervals and order quantities lower than optimal can be attributed to either a misjudgment of the relative importance of the cost components and/or to a need for action (i.e., placing an order). Furthermore, the non-optimal adjustment of $(O Q, O T)$ can be explained, as previously, by a reluctance of subject to take the risk of shipment delay (i.e., backorder cost). Note that the replenishment policy leading to the lowest possible total cost requires values of $(O Q, O T)$ that are too low to be practically implemented. They are significantly lower than the market ones.

The analysis of the results of variability of order quantity and order time intervals suggests that there is a range of low, medium, and high variability on the subjects' decision indicators. Based on the observations in Table 4, the majority of retailers (67\%) have medium variability of their replenishment decision indicators. Wholesalers behave differently. $17 \%$ of wholesalers have low variability of their replenishment indicators, whereas $33 \%$ of them introduce medium variability in their ordering behavior. Some of the distributors $(25 \%)$ exhibit a medium variability, whereas the majority of them introduce high variability in their replenishment decision indicators. Although there are three subjects (R12, W10, D10) who follow the fixed order quantity and variable order time intervals ordering behavior, the majority of the subjects (91\%) have variable order quantity and variable order time intervals ordering behavior. Furthermore, on the echelon level, results show that retailer has low variability on order quantity and medium variability on order time intervals. Wholesaler shows medium variability on both order quantity and order time intervals. Distributor has a medium variability on order quantity and high variability on order time intervals. Hence, results from the both individual and echelon levels confirm that the variability of order quantity and order time intervals tends to increase while moving upstream from the market along the supply chain.

One interesting point remains to be discussed further: why do wholesalers behave differently? Prior work (e.g., Baganha and Cohen, 1998; Blinder 1981; Cachon et al., 2007; Rong et al., 2008; West, 1986) has highlighted that the variability of order quantity increases from retailer to wholesaler and decreases from wholesaler to manufacturer. This study confirms the difference in wholesaler ordering behavior, but it distinguishes from previous works since we observe a constant increase in the variability of order quantity, but a decrease in the variability of the wholesaler order time intervals at the echelon level. Moreover, at the individual level 
analysis, half of wholesalers (50\%) in Table 3 and Table 4 are placed on the low and medium variability classification. These evidences, in connection with the previous discussion, reveal that wholesalers inject variability in order quantity and smoothens order time intervals under continuous review. Therefore, wholesalers perform a smoothing role in the middle of supply chain.

Subjects hold on-hand inventory to guard against unknown customer orders and unreliable supplier shipment. Since there is no price variation in our study, the main reason to hold onhand inventory is to avoid stock-out and incurring backorder costs. The higher variability of the order quantity and order time intervals may lead to higher costs for both inventory manager and his/her supplier. Inventory managers' on-hand inventory depends on the on-hand inventory availability at the upstream supplier, and on the time delays for ordering and shipment. As shown in Table 7, larger order quantities and longer order time intervals increase inventory manager holding and backorder costs. The other important point is that replenishment policy of the most upstream echelon is decisive regarding the total cost of supply chain (see Tables 5 and 6). The larger order quantity of distributor, the larger total costs of wholesaler and retailer. This observation also holds for distributor's lengthened order time intervals.

Results in Table 8 suggest that a reduction in the variability of order quantity specifically for upstream echelons could help the distributor to cut unnecessary backorder and holding costs. Costs are more correlated to upstream echelons due to the higher variations of replenishment decision indicators. This observation is important, as it was somewhat unexpected. Indeed, the literature usually looks downstream for the source of variability and fluctuations. Results in Table 9 show that the high variability of wholesaler replenishment decision indicators increases distributor backorder cost. Also, high variability of retailer order quantity increases wholesaler holding cost. Therefore, results suggest that reduction in the variability of customer order quantity significantly decreases the supplier total cost.

\section{Conclusion}

This research examines the behavior of inventory managers under continuous review in decentralized supply chain. A specific experiment is designed to control the environment and avoid possible perturbations. The experimental setting is characterized by unknown market demand and local information availability. This research contributes to inventory replenishment literature by revealing $(i)$ the change in order time intervals along the supply chain, $(i i)$ the smoothing role of wholesalers in the decentralized supply chain, (iii) the influence of inventory managers' replenishment decisions on their own and the other echelons' costs.

This work purposely focuses on individual and echelon levels. One possible extension would be studying the aggregated supply chain behavior. This study uses student subjects; this should not constitute a limitation according to (e.g., Croson and Donohue, 2006; Holweg and Bicheno, 2002) who report no significant difference between students and professional subjects. Nevertheless, the authors have experienced situations in which professional do tend to behave differently from students. Thus, it would be worth investigating potential deviations between these two categories.

A normative model, along with optimization procedures, and using the same set of decision variables as in the experiment is implemented to serve as a reference for the evaluation of human decision behavior. It is observed that human replenishment decisions are not perfectly 
optimal. The difference indicates a risk reluctance that leads to inventory larger than optimal. Consequently, human decision makers do not reach the optimal balance between back-order cost and inventory carrying cost.

Future research in this area may examine the robustness of this study's results in different supply chain contexts and with a larger number of teams. Different experimental settings such as known demand to remove demand signal processing from structural causes of order amplification could help understanding the behavioral factors under continuous review. The experimental platform used in this research is richer in many ways than the computerized beer game. It allows us to systematically study the manager inventory replenishment decisions under continuous review. Thus, allowing communication and information sharing between supply chain members in the experiment could also extend this work. 


\section{References}

[1] Arguilaget Montarelo, L., Glardon, R., Zufferey, N. 2017. A Global SimulationOptimization Approach for Inventory Management in a Decentralized Supply Chain, Supply Chain Forum: An International Journal, 18 (2), 112-119.

[2] Aviv, Y. 2002. A Time Series Framework for Supply Chain Inventory Management. Operations Research, 51, 210-227.

[3] Badinelli, R., D. 1992. A Model for Continuous-Review Pull Policies in Serial Inventory Systems. Operations Research, 40, 142-156.

[4] Baganha, M. P., Cohen, M. A. 1998. The stabilizing effect of inventory in supply chains. Operations Research, 46 (3), 72-83.

[5] Bendoly, E., Croson, R., Goncalves, P., Schultz, K. 2009. Bodies of Knowledge for Research in Behavioral Operations. Production and Operations Management, Volume 19, 434-452.

[6] Bendoly, E., Donohue, K., Schultz, K. 2006. Behavior in Operations Management: Assessing recent findings and revisiting old assumptions. Journal of Operations Management, 24, 737-752.

[7] Blinder, A.S. 1981. Retail inventory behavior and business fluctuations, Brooking Papers on Economic Activity, 2, 443-520.

[8] Bolton, G. E. Katok, E. 2005. Learning-by-doing in the newsvendor problem. Discussion paper, Penn State University.

[9] Cachon, G. P., Randall, T., Schmidt, G. M. 2007. In search of the bullwhip effect. Manufacturing \& Service Operations Management, 9(4), 457-479.

[10] Carle, M.-A., Martel, A., Zufferey, N. 2012. The CAT Metaheuristic for the Solution of Multi-Period Activity-Based Supply Chain Network Design Problems, International Journal of Production Economics 139 (2), 664-677.

[11] Chatfield, D. C., Kim, J. G., Harrison, T. P., Hayya, J. C. 2004. The bullwhip effectimpact of stochastic lead time, information quality, and information sharing: A simulation study. Production and Operations Management, 13(4), 340-353.

[12] Chen, F. 1999. Decentralized supply chains subject to information delays. Management Science, 45 (8), 1076-1090.

[13] Clark, A. Scarf, H. 1960, Optimal Policies for a Multi-Echelon Inventory Problem, Management Science, 6, 475- 490.

[14] Croson, R., Donohue, K. 2002. Experimental Economics in Supply Chain Management: An Experimental Study. Interfaces, 32, 74-82.

[15] Croson, R., Donohue, K. 2003. Impact of POS data sharing on supply chain management: an experimental study. Production and Operations Management, 12, 111.

[16] Croson, R., Donohue, K. 2006. Behavioral causes of the bullwhip effect and the observed value of inventory information. Management Science, 52, 323-336.

[17] Croson, R., Donohue, K., Katok, E., Sterman, L.D. 2012. Order Stability in Supply Chains: Coordination Risk and the Role of Coordination Stock. In Working Paper, Smeal College of Business, Penn State. State College, PA.

[18] De Bodt, M. A., Graves. S. C. 1985. Continuous-Review Policies for a Multi-Echelon Inventory Problem with Stochastic Demand. Management Science, 31, 1286-1299.

[19] Dogan, G. Sterman, J.D. 2005. When less leads to more: Phantom ordering in the beer game. Proceedings of the 2005 International System Dynamics Conference, Boston, MA. 
[20] Dogan, G. Sterman, J.D. 2006. I'm not hoarding, I'm just stocking up before the hoarders get here Behavioral causes of phantom ordering in supply chains, MIT Sloan School of Management, Working paper.

[21] Federgruen, A., Zipkin, P. 1984. An efficient algorithm for computing optimal (s, S) policies. Operations Research, 32(6), 1268-1285.

[22] Forrester, J. W. 1961. Industrial dynamics (Vol. 2). Cambridge, MA: MIT press.

[23] Gino, F., Pisano, G. 2008. Toward a Theory of Behavioral Operations. Manufacturing \& Service Operations Management, 10(4), 676-752.

[24] Grubbström, R. W., Wikner, J. 1996. Inventory trigger control policies developed in terms of control theory. International Journal of Production Economics, 45 (1), 397 406.

[25] Gupta, S., Steckel, J. and Banerji, A. 2001. Dynamic decision making in marketing channels: An experimental study of cycle time, shared information and customer demand patterns (A. Rapoport, R. Zwick, Eds.). Experimental Business Research. Kluwer Academic Publishers, Boston, MA.

[26] Holweg, M., Bicheno, J. 2002. Supply chain simulation - a tool for education, enhancement and endeavor. International Journal of Production Economics, 78 (2), 163-175.

[27] Kaminsky, P., Simchi-Levi, D. 1998. A new computerized beer game: A tool for teaching the value of integrated supply chain management. Global Supply Chain and Technology Management (Hau Lee and Shu Ming Ng, Eds.). POMS Series in Technology and Operations Management, 216-225.

[28] Katok, E., 2011. Using laboratory experiments to build better operations management models. Foundations and Trends in Technology, Information, and Operations Management, 5 (1), 1-86.

[29] Kimbrough, S. O., Wu, D. J., Zhong, F. 2002. Computers play the beer game: can artificial agents manage supply chains?. Decision support systems, 33(3), 323-333.

[30] Lee, H. L., Billington, C. 1993. Material management in decentralized supply chains. Operations research, 41(5), 835-847.

[31] Lee, H. L., Padmanabhan, V., Whang, S. 1997. Information distortion in a supply chain: the bullwhip effect. Management Science, 43(4), 546-558.

[32] Loch, C. H., Wu, Y. 2007. Behavioral Operations Management, Operations Management, vol. 1, no. 3, pp 121-232.

[33] Machuca, J.A.D., Barajas, R.P. 2004. The impact of electronic data interchange on reducing the bullwhip effect and supply chain inventory costs. Transportation Research Part E, 40, 209-228.

[34] Montreuil, B., Brotherton, E., Glardon, R., Yoo, M, J., Elamiri, Y., Borter, A., Morneau, A., Naciri, S. Jermann, P. 2008. Experiences in Using XBeer Game Virtual Gaming for Learning Supply Chain Management, 2nd European Conference on Games Based Learning, Which city, Spain, 16-17 October.

[35] Oliva, R., Gonçalves, P. 2007. Behavioral Causes of the Bullwhip Effect: "Satisficing" Policies with Limited Information Cues. Under revision for resubmission to Journal of Operations Management.

[36] Respen, J., Zufferey, N., Wieser, Ph. 2017. Three-level inventory deployment for a luxury watch company facing various perturbations, Journal of the Operational Research Society, 68 (10), $1195-1210$.

[37] Rong, Y., Shen, Z., J., M., Snyder, L., V. 2008. The Impact of Ordering Behavior on Order-quantity. Variability: A Study of Forward and Reverse. Bullwhip Effects. Flexible Services and Manufacturing Journal, 20, 95-124. 
[38] Schmidt, C. P., Nahmias, S. 1985. Optimal policy for a two-stage assembly system under random demand. Operations Research, 33 (5), 1130-1145.

[39] Schweitzer, M. E., Cachon, G. P. 2000. Decision bias in the newsvendor problem with a known demand distribution: Experimental evidence. Management Science, 46 (3), 404-420.

[40] Silver, E. A., Zufferey, N. 2011. Inventory Control of an Item with a Probabilistic Replenishment Lead Time and a Known Supplier Shutdown Period, International Journal of Production Research 49 (4), 923-947

[41] Simon, H. A. 1952. On the application of servomechanism theory in the study of production control. Econometrica: Journal of the Econometric Society, 247-268.

[42] Simon, H. A. 1969. The Sciences of the Artificial. The MIT Press, Cambridge.

[43] Spearman, C. 1904. The proof and measurement of association between two things. The American Journal of Psychology, 15 (1), 72-101.

[44] Steckel, J.H., Gupta, S., Banerji, A. 2004. Supply chain decision making: will shorter cycle times and shared point-of sale information necessarily help. Management Science, 50, 458-464.

[45] Sterman, J. D. 2000. Business dynamics: systems thinking and modeling for a complex world (Vol. 19). New York: Irwin/McGraw-Hill.

[46] Sterman, J.D. 1989. Modeling of Managerial Behavior: misperceptions of feedback in a dynamic decision making experiment. Management Science, 35, 321-339.

[47] Thevenin, S., Zufferey, N., Widmer, M. 2015. Metaheuristics for a Scheduling Problem with Rejection and Tardiness Penalties, Journal of Scheduling 18 (1), 89-105.

[48] Thevenin, S., Zufferey, N., Widmer, M. 2016. Order acceptance and scheduling with earliness and tardiness penalties, Journal of Heuristics 22 (6), 849-890.

[49] Towill, D. R. 1991. Supply chain dynamics. International Journal of Computer Intergrated Manufacturing, 4, 197-208.

[50] Vassian, H. J.1955. Application of discrete variable servo theory to inventory control. Journal of the Operations Research Society of America, 3, 272-282.

[51] Voldrich, S., Wieser, Ph., Zufferey, N. 2017. Competitive and Timely Food Supply combined with Operational Risk, Supply Chain Forum: An International Journal, 18 (1), 2-6.

[52] West, K. D. 1986. A Variance Bound Test of the Linear Quadratic Inventory Model. Journal of Political Economics, 94 (2), 374- 401.

[53] Wikner, J. Towill, D.R. Naim. M. 1991. Smoothing supply chain dynamics. International Journal of Production Economics, 22, 231-248.

[54] Wu, D.,Y. Katok, E. 2006. Learning, communication, and the bullwhip effect. Journal of Operations Management, 24, 839-850. 